\title{
An analysis of solar energy conversion systems based on photon and thermal processes
}

\author{
Jolanta Fieducik ${ }^{1}$, Jan Godlewski ${ }^{2}$ \\ ${ }^{1}$ University of Warmia and Mazury in Olsztyn, Faculty of Technical Sciences; \\ ul. Oczapowskiego 11,10-749 Olsztyn,Poland; email: joalnta.fieducik@uwm.edu.pl \\ ${ }^{2}$ Gdańsk University of Technology, Faculty of Applied Physics and Mathematics; \\ ul. Gabriela Narutowicza 11/12, 80-233 Gdańsk, Poland
}

(C) 2016 Authors. This is an open access publication, which can be used, distributed and reproduced in any medium according to the Creative Commons CC-BY 4.0 License requiring that the original work has been properly cited.

Received: 2016-10-28; accepted: 2017-02-23

\begin{abstract}
Solar spectral irradiance covers a fairly broad wavelength range. Solar radiation is part of the electromagnetic spectrum which is described by the concept of wave-particle duality. The corpuscular theory of electromagnetic radiation states that energy is transmitted by photons. Photons carry specific amounts of energy which can be used to convert solar energy into other types of energy, in particular electricity.

The internal photoelectric effect is important for the conversion of solar energy to electricity because the efficiency of the external photoelectric effect is too low. Every quantum of solar energy also carries a certain amount of energy which can be converted into heat energy.

The objective of the paper is to analyze and compare the efficiency of solar energy conversion in photon and thermal processes, to review the technological advances made in this field, and to indicate potential directions for the development of systems converting solar energy to electricity.
\end{abstract}

Keywords: energy conversion, photoelectric effect, thermoelectric process, thermionic emission process

\section{INTRODUCTION}

Direct conversion of solar energy is an important aspect in the generation of renewable energy. The main emphasis is on solutions that directly convert solar energy to electricity, and these types of applications will be discussed in this paper.

Solar energy can be converted to electricity by relying on the particle nature or wave nature of radiation. In processes that rely on particles of solar energy, a single quantum is converted into a specific amount of electrical energy. Conversion processes that rely on the energy of single photons involve external and internal photoelectric effects. The internal photoelectric effect is widely used in photovoltaic cells, whereas the external photoelectric process has very few practical applications.
The wave nature of solar radiation does not have discrete properties which characterize the particle nature of light. Regardless of wavelength, the energy carried by solar radiation can always be converted to thermal energy and, consequently, to electricity. Solar thermal energy is directly converted to electricity in a thermoelectric process which involves thermionic emission or a combination of various phenomena. Solar thermal energy can be converted to electricity in mechanical and thermodynamic systems, but solutions of this type will not be reviewed in this paper.

Photon and thermal processes involved in the direct conversion of solar radiation to electricity will be discussed to analyze the potential efficiency of energy conversion systems and the technological progress made in this field. 
The discussed subject attracted the researchers' interest because the thermal processes involved in the conversion of solar energy are characterized by higher efficiency than photon processes and could play a leading role in the conversion of solar energy to electricity in the future

\section{ENERGY CARRIED BY SOLAR RADIATION}

The energy carried by solar radiation is illustrated in Figure 1 (Thekaekara 1976). The diagram in Figure 1 features three curves. One of them describes the spectral irradiance of solar radiation outside the Earth's atmosphere. When irradiance is summed up in all wavelengths, the result will be the overall energy carried by solar radiation outside the Earth's atmosphere. The radiation flux is estimated at $1360 \mathrm{~W} / \mathrm{m}^{2}$, and it is referred to as the solar constant. Solar energy can be summed up in stages, from the shortest to longer wavelengths. The resulting curve is presented in Figure 1, and it illustrates the part of the total radiation flux, i.e. the solar constant, which covers different wavelengths. The curve demonstrates that nearly the whole of the solar electromagnetic radiation is found below the wavelength band of 2 micrometers. The diagram in Figure 1 also illustrates the theoretical distribution of electromagnetic radiation emitted by a blackbody in a thermal equilibrium at a temperature of $6050 \mathrm{~K}$ according to Planck's law.
The energy flux of the spectral irradiance of solar radiation reaching the Earth's surface is somewhat smaller than the energy flux reaching the upper limit of the atmosphere because the solar beam is weakened as it crosses the atmosphere. As a result, the radiation flux incident on a plane perpendicular to the solar rays depends on the Earth position and for AM1.5G is standardized at $1000 \mathrm{~W} / \mathrm{m}^{2}$ as compared to $1360 \mathrm{~W} / \mathrm{m}_{2}$ before it enters the atmosphere. Detailed reasons for changes in the energy flux of spectral irradiance in the atmosphere will not be discussed in this paper and can be found in the existing literature (Thekaekara 1976, PV Measurements, on-line).

High-efficiency solar energy harvesters have to convert the greatest possible amount of solar radiation reaching the Earth's surface into usable energy. A converter that transforms nearly all of the available solar energy into usable energy, such as electrical energy, would be the ideal solution. The most advanced solar energy conversion systems rely on photon processes in photovoltaic diodes. Considerable progress has also been made in conversion systems that rely on thermoelectric phenomena, thermionic emission and photoemission. The efficiency of photon, thermoelectric and thermionic emission processes in converting solar energy to electricity will be analyzed in successive sections of this paper. Their potential and practical applications in future solutions will also be discussed.
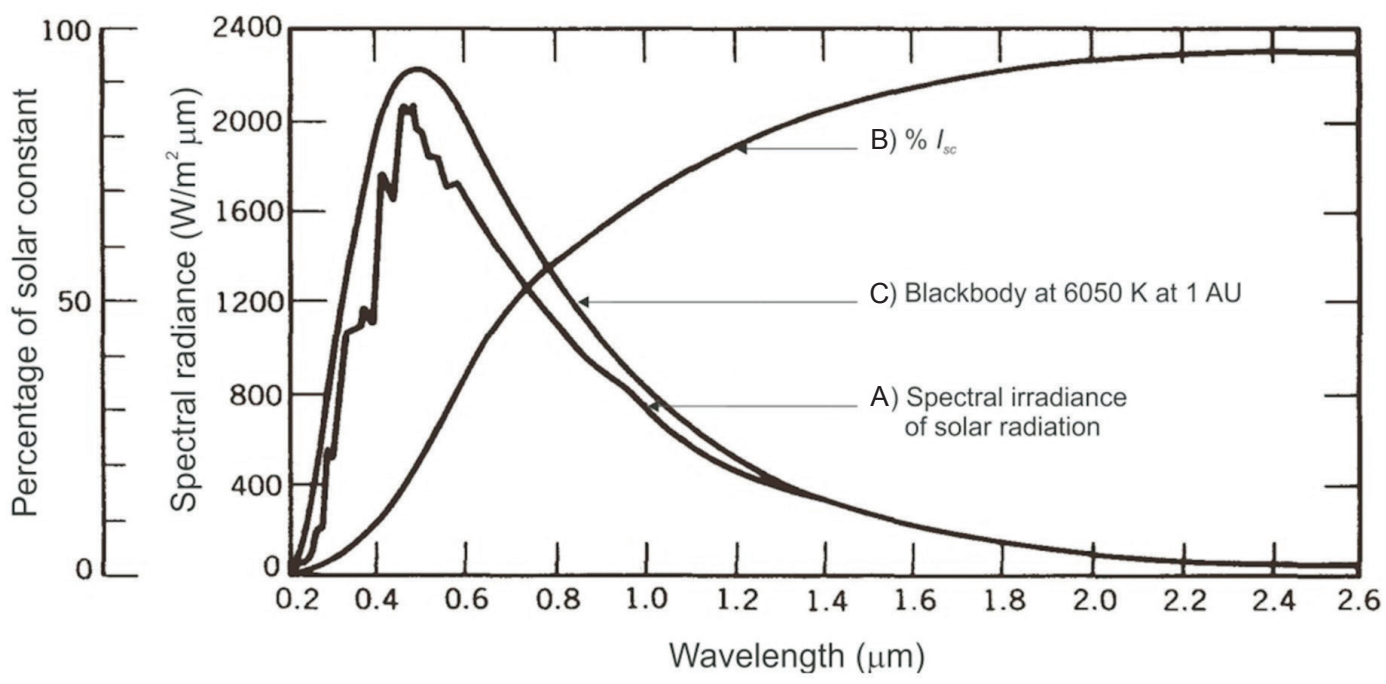

Fig. 1. Spectral irradiance of solar radiation outside the Earth's atmosphere (A); radiation flux as a function of wavelength, counting from the shortest waves (B); distribution of electromagnetic radiation emitted by a blackbody at a temperature of 6050 K according to Planck's law (C) (Thekaekara 1976) 


\section{PHOTON PROCESSES OF CONVERTING SOLAR ENERGY TO ELECTRICITY}

In photon processes, the amount of solar energy that can be converted to electricity is limited by the amount of energy carried by a photon. In an internal photoelectric process, this limit is denoted by the band gap in a semiconductor (Peters et al. 2010, Congreve et al. 2013), in organic materials with exciton binding energy (De Vos 1980, Sze \& Ng 2006), whereas in an external photoelectric process - by the work function of the material. In every process, the conversion of photon energy to electricity is most efficient for photons whose energy is somewhat higher than threshold values, and this efficiency decreases with an increase in photon energy. To date, the efficiency of systems that convert solar energy to electricity by relying on the external photoelectric effect or organic photovoltaic cells has never exceeded $12 \%$. The methods that can be used to increase the efficiency of such systems will not be discussed in this paper. The potential applications of the internal photoelectric effect in inorganic semiconductors, which contributes to relatively high conversion efficien$c y$, will be evaluated in this section. At present, the conversion efficiency of multi-junction photovoltaic cells reaches $46 \%$ under laboratory conditions (a triple-junction cell developed by Soitec and the Fraunhofer Institute for Solar Energy Systems). Such high conversion efficiency was achieved by relying on a broad wavelength range of solar radiation. Multi-junction cells were selected to utilize the broadest possible spectrum of solar radiation. The spectral efficiency of a triple-junction photovoltaic cell is illustrated in Figure 2.

The spectral efficiency of the photovoltaic cell presented in Figure 2, which absorbs solar radiation in the wavelength range of $300 \mathrm{~nm}$ to $1800 \mathrm{~nm}$ via three cells aligned parallel to the Sun's rays. The spectral range represented by spectral efficiency curves in Figure 1 contains nearly $90 \%$ of solar energy. For this reason, and due to the highly efficient processes of converting photon energy in broadband multi-junction photovoltaic cells, experimental conversion efficiency reaches $46 \%$. The theoretical efficiency of photovoltaic cells containing an infinite number of junctions is estimated at $68 \%$ under exposure to non-concentrated sunlight and $87 \%$ under exposure to concentrated sunlight (Shockley \& Queisser 1961). However, such values are unlikely to be achieved in practice, even in the distant future, due to significant energy losses.

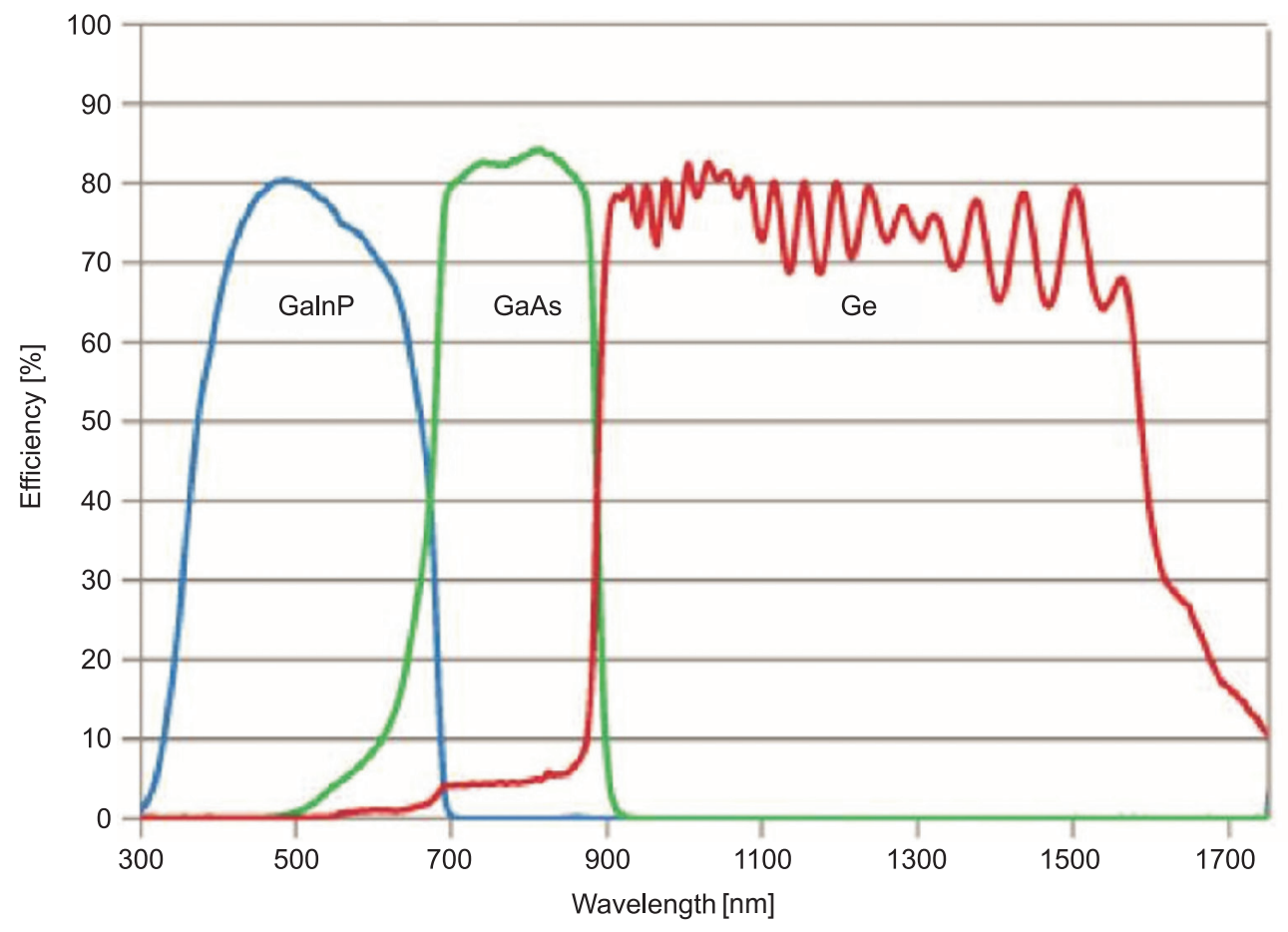

Fig. 2. Spectral efficiency of a GaInP/GaAs/Ge triple-junction photovoltaic cell (Marius et al. 2010) 
In single-layer cells, the maximum theoretical efficiency of sunlight to electric energy conversion is estimated at $33.7 \%$ under exposure to the AM 1.5 spectrum and the optimal bandgap of $1.37 \mathrm{eV}$ (PV Measurements, on-line). The efficiency of single-layer cells made of crystalline silicon reached $25 \%$ under laboratory conditions (Pope \& Swenberg 1999). Multi-junction cells are more expensive than single-layer cells, and they are cost-efficient only when photovoltaic cells have a small surface area. This can be achieved by concentrating the sunlight reaching the solar panel. The above values were given as a general representation of the conversion efficiency of photovoltaic cells.

\section{THERMAL PROCESSES OF CONVERTING SOLAR ENERGY TO ELECTRICITY}

An analysis of processes during which solar energy is converted to other types of energy should account for the fact that solar radiation can be transformed into heat energy over the entire spectrum. The whole of the energy carried by solar radiation is captured in the process of converting sunlight to thermal energy but efficient conversion of the absorbed heat to electricity poses the main problem. The latest technological advances indicate that thermal energy can be directly converted to electricity with very high efficiency. At present, processes based on thermal energy can compete with photon processes which are involved in the conversion of solar energy to electricity as part of the internal photoelectric effect. Those processes will be discussed in greater detail in successive parts of this section.

Thermal energy can be effectively converted to electricity in a thermoelectric process (Seebeck effect) and a thermionic emission process. Systems that convert heat to electricity are shown in the diagrams below. A diagram of a solar thermoelectric generator is presented in Figure 3, and a thermionic generator is shown in Figure 4.

In a thermoelectric generator, the conversion of thermal energy to electricity relies on the Seebeck effect, and in a thermionic generator - on the process of thermionic emission from materials with a high temperature.

When thermal energy captured from solar radiation is converted into any type of energy, the maximum energy conversion efficiency is expressed by the following formula (Pope \& Swenberg 1999, Quintana et al. 2013):

$\eta_{h}=\left(1-\frac{\sigma \cdot T_{R}^{4}}{P_{\text {sun }}}\right)\left(1-\frac{T_{\text {amb }}}{T_{R}}\right)$

where $T_{R}$ is receiver temperature, $T_{\text {amb }}$ is ambient temperature, $P_{\text {sun }}$ is the energy flux absorbed from solar radiation, and $\sigma$ is the Stefan-Boltzmann constant.

Expression (1) has two terms. The first is determined by the loss of energy from a heat receiver due to thermal radiation, and the second follows from the second law of thermodynamics. The two terms determine the efficiency of thermal energy conversion to electricity. Thermal radiation of the heat receiver, warmed by solar radiation, plays an important role in the conversion process.

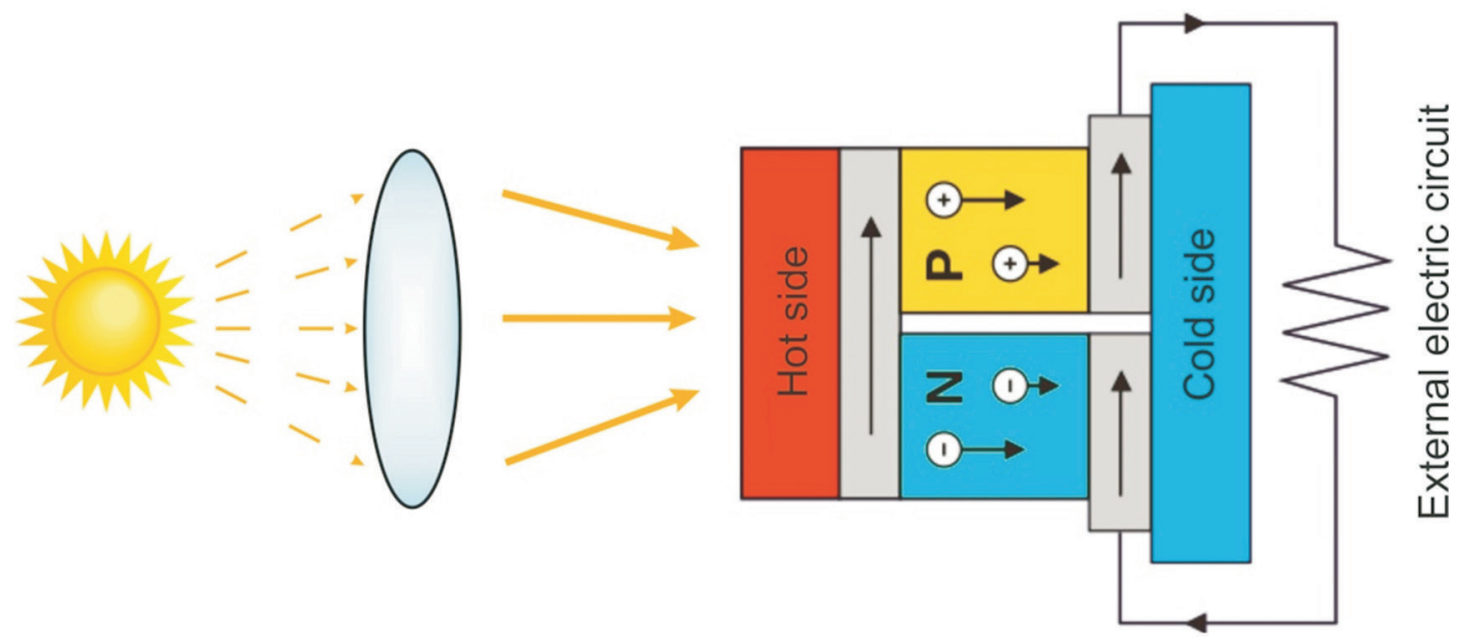

Fig. 3. Diagram of a solar thermoelectric generator (adapted from Bell 2008) 


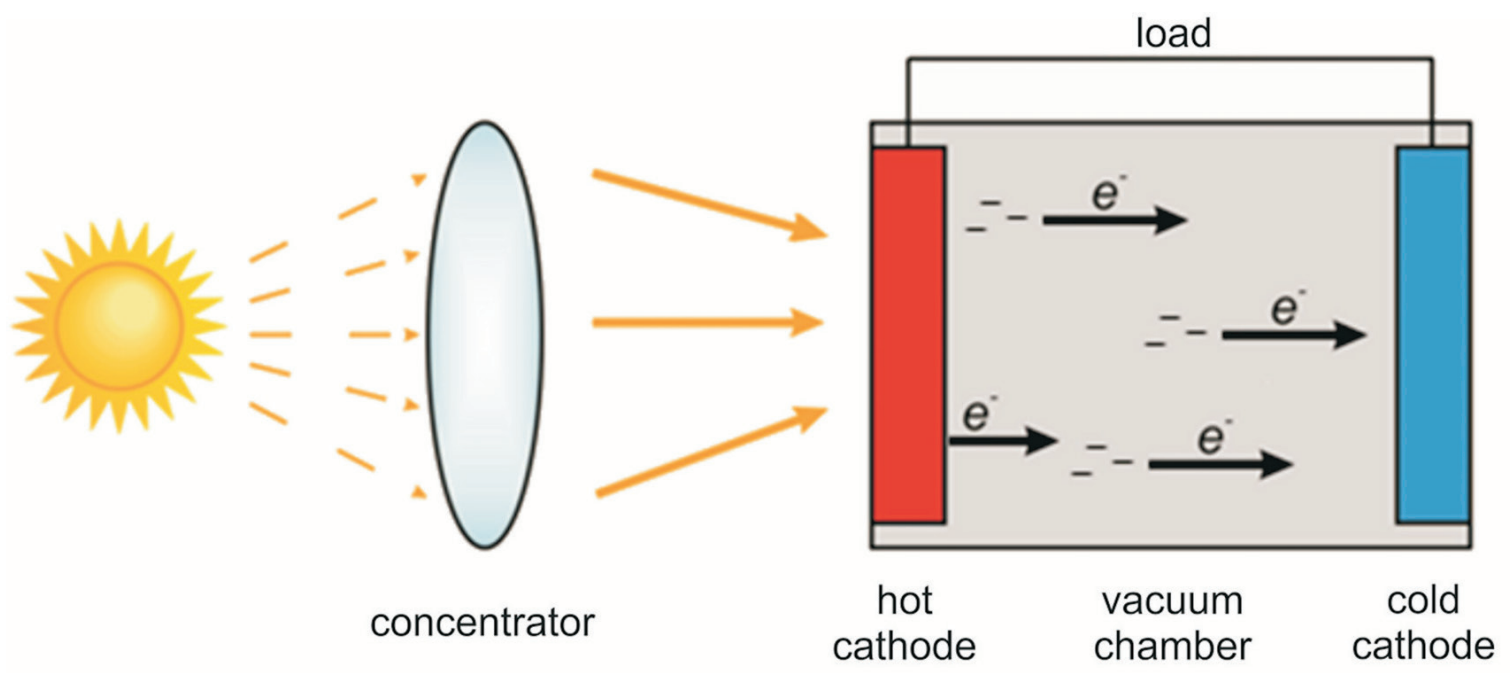

Fig. 4. Conversion of solar energy to electricity by thermionic emission (adapted from Meir et al. 2013)

The term which follows from the second law of thermodynamics causes conversion efficiency to increase with a rise in receiver temperature, whereas the first term in formula (1) has the opposite effect. The two terms produce extreme values of conversion efficiency as a function of temperature. The selection of an optimal temperature for specific system parameters significantly increases conversion efficiency. For example, in an ideal environment with a concentration factor of $1000 \times$ and ambient temperature of $300 \mathrm{~K}$, maximum conversion efficiency is achieved when the temperature of the receiver is $1107 \mathrm{~K}$. Under the above conditions, conversion efficiency is $66.7 \%$, which is extremely high for a process where thermal energy is converted to electricity or other types of energy.

The above efficiency could be achieved if solar energy could be converted to electricity in its entirety. In practical applications, efficiency is defined as electric energy generated through the load circuit from thermal energy absorbed by the hot junction. In this case, efficiency is determined by the electrical parameters of the energy converter.

In a thermoelectric process (Fig. 3), the maximum energy conversion efficiency is expressed by the following formula (Meir et al. 2013, Bell 2008):

$\eta_{\max }=\frac{T_{H}-T_{C}}{T_{H}} \frac{\sqrt{1+Z \bar{T}}-1}{\sqrt{1+Z \bar{T}}+\frac{T_{C}}{T_{H}}}$ where $Z \bar{T}$ is determined by the properties of the material in the Seebeck effect and is expressed as:

$$
Z \bar{T}=\frac{\delta S^{2} T}{\lambda}
$$

In the above formulas, $T_{H}$ is the temperature of the hot junction, $T_{C}$ is the temperature of the cold junction, $\delta$ is the electrical conductivity of a material with Seebeck coefficient $S, \lambda$ is the thermal conductivity of a material, and $T$ is the average temperature of that material. The typical value of $Z \bar{T}$ in materials used in thermoelectric processes ranges from 1 to 3 , but it can be much higher for some materials, such as graphene which has a $Z \bar{T}$ of 20 (Schwede et al. 2010).

The efficiency of thermal energy conversion in thermoelectric processes increases with technological progress. At present, energy conversion efficiency in a thermoelectric process is not very high and ranges from $5 \%$ to $10 \%$, but values up to $30 \%$ are expected in the near future (Baranowski et al. 2012).

In the Seebeck effect, electricity is generated when a temperature gradient applied in a semiconductor causes charge carriers to diffuse. A completely different mechanism is involved in the generation of electricity in thermionic emission. In this process, electricity is produced when a hot cathode emits electrons into the vacuum (Fig. 4). The electrons emitted by a thermionic cathode have energy which generates electricity in a circuit. Total current density resulting from 
thermionic emission is given by the RichardsonDushman equation:

$$
J=A T^{2} \exp \left(-\frac{\phi}{k T}\right)
$$

where $A$ is the Richardson constant, $T$ is temperature in degrees Kelvin, $\phi$ is the work function of the cathode material, and $k$ is the Boltzmann constant. The above formula indicates that the emitted electron current will increase with a rise in the temperature of the emitter. The work function of the emitter plays a very important role because it has an exponential effect on current density. The production of a stable electron emitter with a low work function is a technological challenge in this field of research.

A formula describing the current-voltage characteristics and the efficiency of thermal energy conversion to electricity for a thermionic diode is more complex because current is strongly dependent on voltage and because current flowing through the diode is limited by spatial charge. Various methods have been proposed for overcoming these technological barriers in the process of designing thermionic diodes that convert thermal energy to electricity (Dragoman \& Dragoman 2007, Bell 2008, Snyter 2008, Schwede et al. 2010, Baranowski et al. 2012, Meir et al. 2013, Segev et al. 2015).

A combination of thermoelectric and photoelectric processes appears to be a highly efficient option for converting solar energy to electricity. This solution is known as photon-enhanced thermionic emission (PETE) which relies on both thermal emission and photoemission of electrons from a cathode to generate electricity in a closed circuit. At present, the conversion efficiency of such systems ranges from $7 \%$ to $15 \%$ (Meir et al. 2013). The efficiency with which solar energy is converted to electricity will continue to increase as new advancements are made in thermionic cathode design. The predicted theoretical energy conversion efficiency of a PETE system is compared with the theoretical efficiency of a single photovoltaic cell in Figure 5 (Meir et al. 2013).

Figure 5 indicates that the combined use of photon and heat fractions of solar radiation increases energy conversion efficiency. The resulting conversion efficiency exceeds the theoretical efficiency of a single photovoltaic diode.

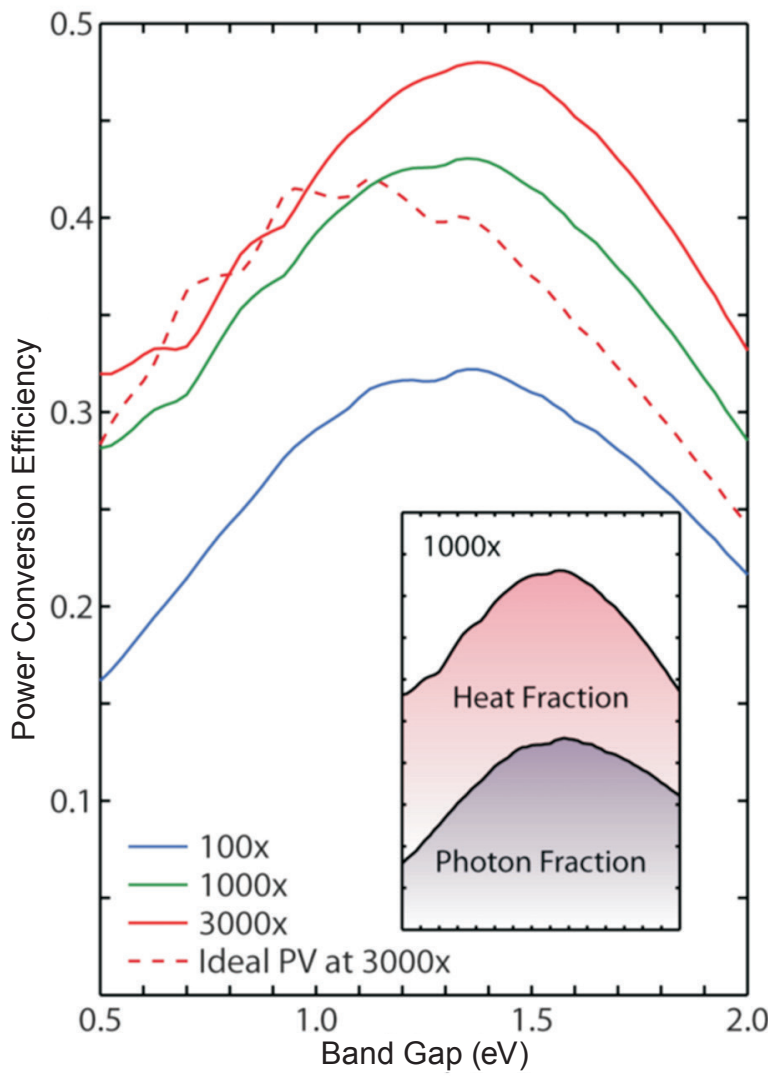

Fig. 5. Relationship between the theoretical power conversion efficiency of photon-enhanced thermionic emission (PETE) at various concentration factors and the work function of cathode material. The dotted line represents the efficiency of an ideal photovoltaic cell with a concentration factor of $3000 \times$. The box inside the chart presents the percentage of the photon fraction and the heat fraction for a thermionic diode with a concentration factor of $1000 \times$

\section{CONCLUSIONS}

The potential methods for directly converting solar energy to electricity have been analyzed. The results indicate that after a period marked by the rapid development of photovoltaic cells, future solutions will rely on electron emission from materials, in particular proton-enhanced thermionic emission (PETE). The development of solar energy harvesters based on PETE requires advancements in thermionic cathode design, in particular with the involvement of nanotubes and fullerenes. Those technologies can be deployed only in concentrated solar power systems. As an additional advantage, these solutions can simultaneously rely on various mechanisms of solar energy conversion, including photovoltaics and thermionic emission, which significantly increases energy conversion efficiency. 


\section{REFERENCES}

Baranowski L., Snyder G. \& Toberer E., 2012. Concentrated solar thermoelectric generators. Energy Environment, 5, 9055-9067.

Bell L.E., 2008. Cooling, Heating, Generation Power, and Recovering Waste Heat with Thermoelectric Systems. Science, 321, 1457-1461.

Congreve D.N., Lee J., Thompson N.J., Hontz E., Yost S.R., Reusswig P.D., Bahlke M.E., Reineke S., Van Voorhis T. \& Baldo M.A., 2013. External Quantum Efficiency Above $100 \%$ in a Singlet-Exciton-Fission-Based Organic Photovoltaic Cell. Science, 340, 334-337.

De Vos A., 1980. Detailed balance limit of the efficiency of tandem solar cells. Journal of Physics D: Applied Physics, $13,839-846$.

Dragoman D. \& Dragoman M., 2007. Giant thermoelectric effect in graphene. Applied Physics Letters, 91, 203116203116-3

Meir S., Stephanos C., Geballe T.H. \& Mannhart J., 2013. Highly-efficient thermoelectronic conversion of solar energy and heat into electric power. Journal of Renewable and Sustainable Energy, 5, 043127-1-043127-15.

Peters M., Goldschmidt J.Ch., Löper P., Groß B., Üpping J., Dimroth F., Wehrspohn R.B. \& Bläsi B., 2010. SpectrallySelective Photonic Structures for PV Applications. Energies, 3(2), 171-193.
Pope M. \& Swenberg Ch.E., 1999. Electronic Processes in Organic Crystals and Polymers. $2^{\text {nd }}$ ed. Oxford University Press.

PV Measurements, http://pvmeasurements.com [access: 10.10. 2016].

Quintana H.A., Song E., Wang G.T. \&. Martinez J.A., 2013. Heat Transport in Novel Nanostructured Materials and their Thermoelectric Applications. Chemical Engineering and Process Techniques, 1, 1-5.

Schwede J.W., Bargatin I., Riley D.C., Hardin B.E., Rosenthal S.J., Sun Y., Schmitt F., Pianetta P., Howe R.T., Shen Zhi-Xun \& Melosh N.A. 2010. Photon-enhanced thermionic emission for solar concentrator systems. Nature Materials, 9, 762-767.

Segev G., Rosenwaks Y. \& Kribus A., 2015. Limit of efficiency for photon-enhanced thermionic emission vs. photovoltaic and thermal conversion. Solar Energy Materials and Solar Cells, 140, 464-476.

Shockley W. \& Queisser H.J., 1961. Detailed Balance Limit of Efficiency of p-n Junction Solar Cells. Journal of Applied Physics, 32, 510-519.

Snyter G.J., 2008. Small Thermoelectric Generators. Electrochemical Society Interface, 17, 3, 54-56.

Sze S.M. \& Ng Kwok K., 2006. Physics of Semiconductor Devices. $3^{\text {rd }}$ ed. Wiley.

Thekaekara M.P., 1976. Solar Radiation Measurement: Techniques and Instrumentation. Solar Energy, 18, 309-325. 